\title{
Collections d'échantillons biologiques humains à visée scientifique. Pourquoi et comment clarifier la réglementation actuelle?
} \author{
ronde $n^{\circ} 7$ de Giens $X X I V^{\star}$ \\ 1 Département de Pharmacologie Médicale, Faculté de Médecine, Lille, France \\ 2 Division Scientifique et Médicale, Pfizer France, Paris, France \\ 3 Agence Française de Sécurité Sanitaire des Produits de Santé, Paris, France \\ 4 Ministère de l'Enseignement Supérieur et de la Recherche, Paris, France
}

Dominique Deplanque ${ }^{1}$, Guillaume Birraux² ${ }^{2}$ Pierre-Henri Bertoye ${ }^{3}$, Éric Postaire ${ }^{4}$ et les participants à la table

Texte reçu le 15 novembre 2008 ; accepté le 20 novembre 2008

\begin{abstract}
Mots clés :
collections biologiques; centres de ressources

biologiques ;

réglementation;

recherche biomédicale

Résumé - La constitution de collections d'échantillons biologiques humains à des fins scientifiques constitue un enjeu majeur en France et en Europe. Dans ce contexte, ces dernières années ont été marquées par la volonté de mettre en place un meilleur encadrement réglementaire. La complexité de celui-ci nécessite cependant quelques éclaircissements. En effet, il convient de préciser la définition de ce qui relève des collections ou des activités de collection biologique, de simplifier les procédures réglementaires de déclaration auprès des Comités de Protection des Personnes (CPP) et du Ministère de l'Enseignement Supérieur et de la Recherche, de discuter de la mise en place d'une méthodologie de référence CNIL (Commission Nationale Informatique et Libertés) dédiée aux modalités particulières de traitement des données associées aux collections biologiques. Il apparait d'autre part indispensable de préciser les modalités d'habilitation et de pérennisation des Centres de Ressources Biologiques, structures dédiées aux activités de collection biologique. Il convient aussi de clarifier le rôle des CPP, notamment dans l'appréciation des modalités d'information et de consentement des sujets au cours de situations particulières comme les collections sur mineurs, les collections avec examen des caractéristiques génétiques ou encore les modalités d'utilisation de collections anciennes. Enfin, en dehors des aspects scientifiques et de santé publique, les collections biologiques d'échantillons humains sont l'objet d'enjeux économiques importants. Dans ce contexte, il est nécessaire d'adapter les procédures permettant par exemple le dépôt de brevets, notamment lorsque plusieurs partenaires publics et/ou privés sont associés. Les prochaines échéances législatives avec la modification du Code de Santé Publique au travers de la loi Jardé et de la révision de la loi de bioéthique permettront peut être de faire valoir ces nécessaires évolutions.
\end{abstract}

Les collections d'échantillons biologiques issus du corps humain constituent un enjeu fondamental, notamment comme support de nombreuses recherches et in fine de progrès diagnostiques et thérapeutiques. Ces dernières années ont été marquées par la volonté d'un meilleur encadrement réglementaire des activités relatives au prélèvement, à la conservation et à l'utilisation des échantillons biologiques issus du corps humain, mais la complexité de la législation française actuelle mérite quelques clarifi-

* Pour la liste des participants, voir en fin d'article. cations voire certaines modifications. À l'occasion de cette table ronde, nous nous sommes ainsi penchés sur plusieurs points relatifs aux collections avec le souci d'une vision pragmatique et la volonté de proposer des aménagements à la fois concrets et consensuels. Nos discussions et propositions ont principalement concerné la définition des activités de collection et les processus de déclaration ou d'autorisation qui y sont attachés, le problème de la mise en place de structures pérennes de conservation (Centres de Ressources Biologiques - CRB), certains aspects 
particuliers de la protection des personnes et enfin les enjeux économiques, scientifiques et de Santé publique, notamment à travers la prise en compte des problèmes liés à la valorisation.

\section{Aspects réglementaires relatifs aux collections biologiques : quelles définition et procédures?}

\subsection{Définition}

La réglementation relative aux collections d'échantillons issus du corps humain pose de nombreuses difficultés, notamment en raison de la multitude de textes qui s'y rapportent et de leur caractère parfois contradictoire. En premier lieu, il existe un problème de définition. La loi de bioéthique de 2004 évoque la notion de «réunion à des fins scientifiques de prélèvements biologiques effectués sur un groupe de personnes identifiées et sélectionnées en fonction des caractéristiques cliniques ou biologiques d'un ou plusieurs membres du groupe, ainsi que des dérivés de ces prélèvements » [article L.1243-3 du Code de Santé Publique (CSP)]. ${ }^{[1]}$ Cependant, au-delà de ces aspects techniques relatifs à la collection, à savoir la conservation, sur le territoire français dans un conteneur et un lieu adaptés, d'échantillons dument sélectionnés, la réglementation précise que l'ensemble des activités de préparation et de conservation d'échantillons issus du corps humain à visée scientifique est concerné. ${ }^{[1]}$ La principale difficulté dans ce contexte est de définir ce qui incombe à l'activité de préparation. Qu'en est-il en particulier de l'activité de prélèvement? Afin de répondre à certaines interrogations soulevées par la loi de 2004, le décret du 10 août 2007 relatif au prélèvement, à la préparation et à la conservation à des fins scientifiques d'éléments du corps humain apporte quelques précisions sur les procédures à respecter selon le contexte de la recherche, en particulier pour ce qui concerne les modalités de déclaration et d'autorisation. ${ }^{[2]}$

\subsection{Diversité des procédures réglementaires}

\section{- Aspects généraux}

De nombreux textes de loi font référence et régissent ces activités. En plus de la loi de Bioéthique (loi n 2004-800 du 6 août 2004), ${ }^{[1]}$ on peut citer les textes encadrant les recherches sur les personnes telles que la loi Huriet révisée par la loi $n^{\circ}$ 2004-806 du 9 août $2004^{[3]}$ et son décret d'application $n^{\circ} 2006-450$ d'avril $2006,{ }^{[4]}$ ou encore la loi informatique et libertés (loi $\mathrm{n}^{\circ} 78-17 \mathrm{du}$ 6 janvier 1978 relative à l'informatique, aux fichiers et aux libertés, modifiée par la loi n 2004-801 du 6 août 2004 relative à la protection des personnes physiques à l'égard des traitements de données à caractère personnel). ${ }^{[5,6]}$ Selon que la recherche soit de type interventionnelle [recherche biomédicale (RBM) ou recherche en soins courants] ou de type non interventionnelle, les modalités de déclaration voire de demande d'autorisation ne seront pas les mêmes (tableau I). ${ }^{[7]}$ Il convient en particulier de distinguer ce qui relève des recherches de type RBM des autres cadres de recherche.

\section{- Cas particulier des recherches de type RBM}

Les recherches de type RBM entrent dans l'un des cadres juridiques des recherches interventionnelles et sont soumises à autorisation auprès de l'Agence française de sécurité sanitaire des produits de santé (Afssaps) ainsi qu'à l'avis d'un Comité de Protection des Personnes. Dans ce cadre particulier, le fait de prélever, de manipuler et de stocker des échantillons d'origine humaine ne constitue pas, aux termes de la loi actuelle, une activité de collection biologique. Les échantillons ou leurs dérivés ne sont considérés comme une collection biologique que s’il est prévu de les conserver à l'issue de la recherche, c'est-à-dire après réalisation de l'ensemble des analyses prévues initialement dans le protocole de recherche. ${ }^{[2-4]}$ Si une telle option est prévue lors de la demande d'autorisation de ce type de recherche, un formulaire spécifique doit être adressé à l'Afssaps conjointement à la demande d'autorisation de la recherche. Cependant, à l'issue de la période couverte par l'autorisation de l'Afssaps, la collection ainsi constituée devra aussi être déclarée au Ministère de l'Enseignement Supérieur et de la Recherche (MESR) comme le sont les collections réalisées sous couvert des autres cadres de recherche. ${ }^{[2-4]}$ Dans ce contexte particulier, la question de l'intérêt et de la pertinence du formulaire Afssaps est clairement posée.

\section{- Autres cadres de recherche}

Dans tous les autres cadres (recherches en soins courants et recherches non interventionnelles), ${ }^{[2-4]}$ les activités de collection biologique doivent faire d'emblée l'objet d'une déclaration auprès du Ministère de l'Enseignement Supérieur et de la Recherche (MESR) et d'un avis auprès du CPP (avis sur l'activité de collection rendu en plus de celui requis pour la recherche elle-même) [tableau I, figure 1]. ${ }^{[7]}$ De plus, si des cessions sont prévues (délivrance d'échantillons à un tiers non impliqué dans le protocole de recherche initial), celles-ci doivent faire l'objet d'une autorisation délivrée par le MESR. Dans le cas de figure où les activités de collection sont regroupées dans un établissement hospitalier, l'Agence Régionale de l'Hospitalisation (ARH) doit aussi être informée. Enfin, lorsque les prélèvements sont réalisés sur des personnes décédées ou concernent des tissus embryonnaires, fœtaux ou des cellules souches embryonnaires, le dossier doit également être soumis à l'Agence de Biomédecine qui a 2 mois pour opposer un éventuel refus. 


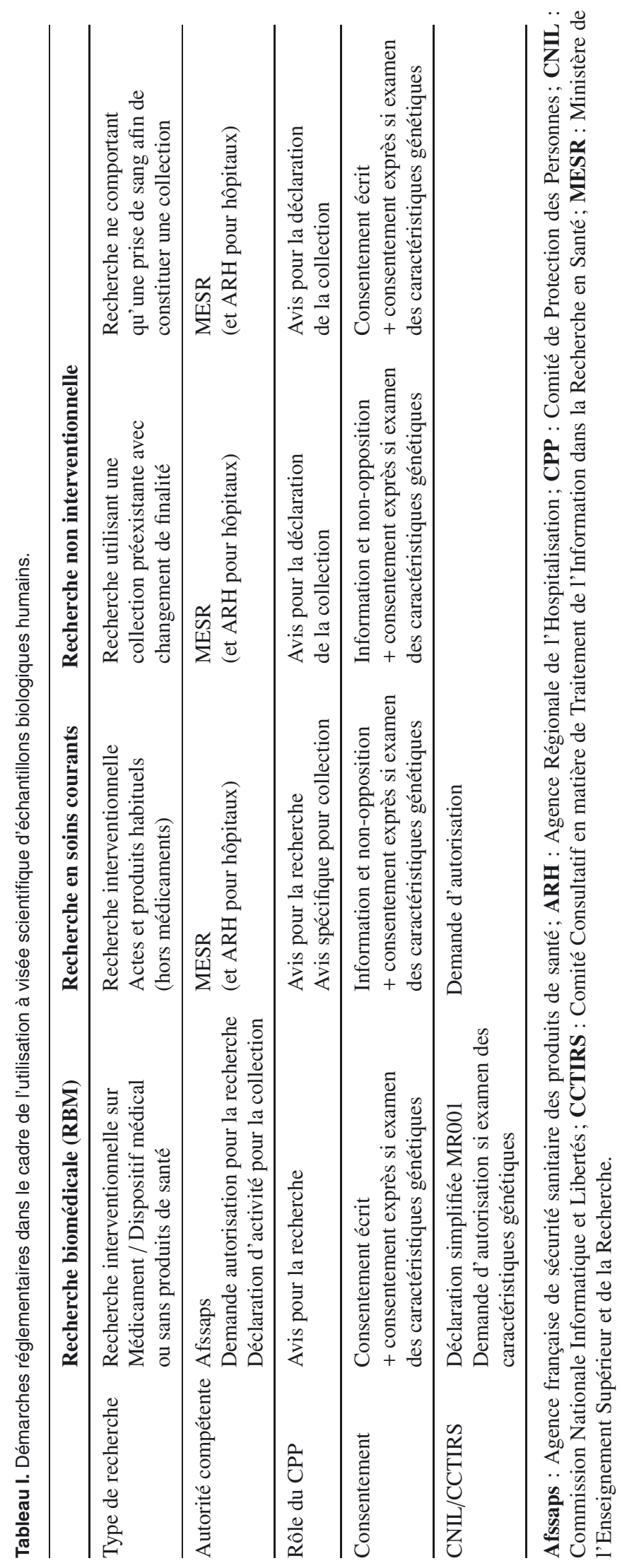




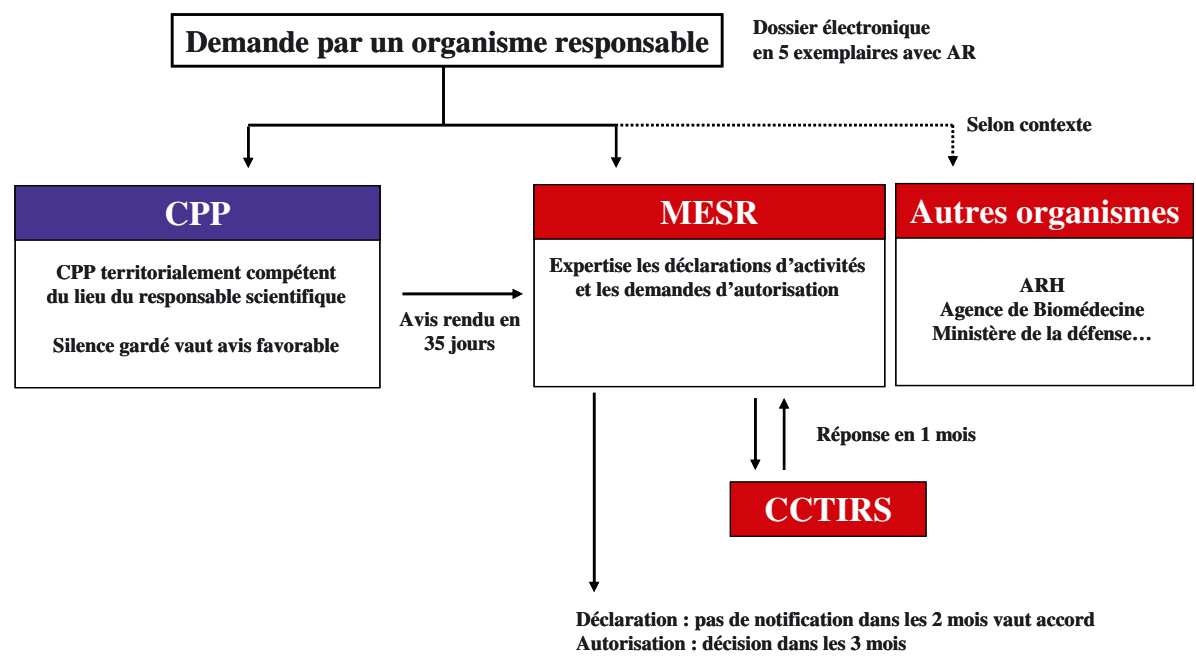

Fig. 1. Schéma de la procédure actuelle d'autorisation d'activités de collection.

CPP : Comité de Protection des Personnes; ARH : Agence Régionale de l'Hospitalisation; AR : Accusé de Réception; MESR : Ministère de l'Enseignement Supérieur et de la Recherche; CCTIRS : Comité Consultatif en matière de Traitement de l'Information dans la Recherche en Santé.

\subsection{Principales difficultés}

De manière générale, la déclaration des activités de collection, quel que soit le cadre réglementaire de la recherche ou de l'activité clinique qui lui est associée, pose de réelles difficultés. En effet, comme nous l'avons déjà souligné précédemment, la déclaration auprès du MESR ne se fait pas de manière individualisée pour chaque collection mais de manière globale par type d'activité déclarée et ce par l'établissement ou l'organisme responsable de la constitution de la collection. Le dossier à compléter précise notamment le type de collection, les responsables et les modalités pratiques de collection (préparation, stockage, traçabilité, locaux, sécurité... .). ${ }^{[7]}$ La préparation de ce dossier demeure une tache difficile, son expertise ne l'étant pas moins ce d'autant que le personnel ministériel impliqué dans l'examen de ces dossiers reste relativement réduit. Les CPP qui ont eux pour mission d'évaluer la qualité de la protection des personnes et de l'information n'ont le plus souvent ni les protocoles des recherches concernées, ni même les documents d'information et/ou de consentement, notamment lorsqu'il s'agit de recherches non interventionnelles. Ces différences sur le contenu des dossiers limitent par ailleurs les interactions entre le MESR et les CPP (figure 1).

Un autre champ de difficultés importantes est celui des autorisations concernant la gestion des données personnelles. La CNIL et le Comité Consultatif en matière de Traitement de l'Information dans la Recherche en Santé (CCTIRS) ont un rôle déterminant qui varie cependant selon le cadre réglementaire de la recherche. ${ }^{[5,6]}$ Dans le cas des RBM, les responsables de la recherche peuvent signer un engagement de conformité à la méthodologie de référence MR-001, référentiel qui définit les modalités de gestion des données dans ce cadre particulier de recherche. ${ }^{[8]}$ Pour l'ensemble des autres cadres réglementaires de recherche, le référentiel MR-001 n'est actuellement pas valide (tableau I). Il convient alors d'appliquer la procédure complète à savoir la demande d'avis auprès du CCTIRS préalable à l'autorisation de la CNIL. ${ }^{[4,6]}$ Le CCTIRS a toute latitude pour émettre un avis négatif sur les modalités de gestion des données à l'occasion du projet de recherche voire sur le projet de recherche lui-même, la CNIL pouvant dans ces conditions refuser de donner son autorisation.

\subsection{Propositions de modifications réglementaires}

- Simplifier les procédures réglementaires

- Suppression dans la loi encadrant les RBM de toute référence aux collections biologiques (loi Jardé entérinée par l'Assemblée Nationale en janvier 2009, en attente d'examen au sénat) $;{ }^{[9]}$

- Simplification de la définition des collections biologiques afin de distinguer plus précisément ce qui relève de la collection d'échantillons biologiques humains de ce qui concerne l'ensemble des activités liées aux collections biologiques;

- Suppression du formulaire Afssaps de déclaration des collections biologiques;

- Séparation des procédures de déclaration au CPP et au MESR. Nous proposons de redéfinir par la loi 2 procédures distinctes, l'une dédiée à la déclaration d'activité de l'organisme responsable, l'autre dédiée à la déclaration des collections (figure 2). Dans ce contexte, le MESR pourrait être impliqué dans la phase initiale de déclaration/autorisation des activités et dans l'habilitation des structures. Une mise à jour périodique (une fois par an) serait ensuite envisagée avec la tenue 


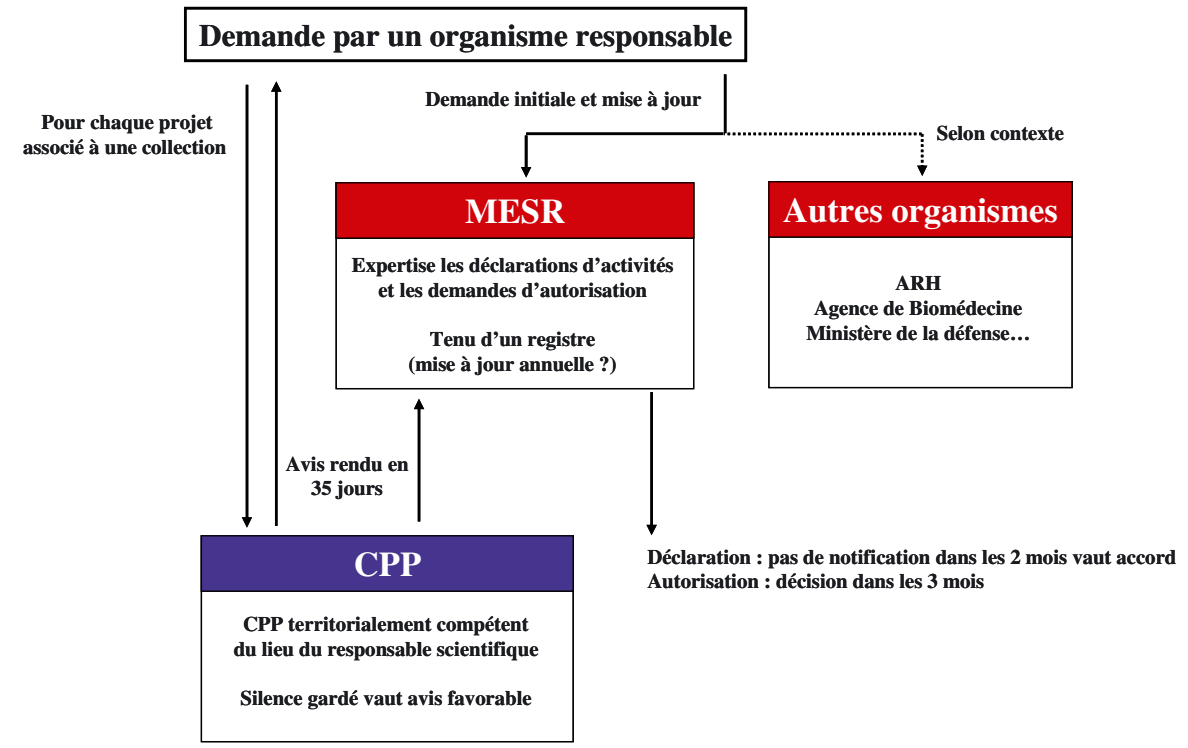

Fig. 2. Proposition d'une nouvelle procédure d'autorisation d'activités de collection.

CPP : Comité de Protection des Personnes; ARH : Agence Régionale de l'Hospitalisation; MESR : Ministère de l'Enseignement Supérieur et de la Recherche.

d'un registre. Les CPP auraient quant à eux la charge d'examiner chaque projet associé à une collection biologique avec un regard particulièrement attentif sur les modalités d'information, de consentement et de protection des personnes.

- Mettre en place une méthodologie de référence CNIL spécifique.

Conformément aux dispositions de l'article 54, alinéa 5 de la loi du 6 janvier 1978 modifiée, ${ }^{[3,5]}$ pour des catégories usuelles de traitements automatisés poursuivant une finalité de recherche et portant sur des données qui ne permettent pas une identification directe des personnes concernées, la CNIL peut, en concertation avec le CCTIRS et les organismes publics et privés représentatifs, homologuer et publier des méthodologies de référence. Si la méthodologie de référence MR-001 couvre tous les traitements de données personnelles mis en œuvre dans le cadre des recherches biomédicales, y compris les essais de pharmacogénétiques, il pourrait être défini un autre référentiel (MR-002 ?) pour les collections biologiques ne permettant pas une identification directe des personnes.

\section{Centres de Ressources Biologiques : outils indispensables à la gestion des collections}

\section{1. État des lieux}

Les structures dédiées à la gestion des collections d'échantillons humains à visée scientifique sont d'une importance capitale pour la recherche médicale. ${ }^{[10,11]}$ En effet, ces structures parfois dénommées Centres de Ressources Biologiques (CRB), interfaces entre les prélèvements de matériel biologique issus de la recherche ou des activités cliniques et les équipes de recherche plus fondamentales, sont devenus des éléments essentiels à de nombreuses recherches. Dans ce contexte, se sont développées des structures relativement spécialisées comme les tumorothèques dont l'objet est la conservation de fragments tumoraux à visée diagnostique et de recherche alors qu'au fil des ans se sont constitués par ailleurs des CRB dont l'objet des collections est une activité de recherche mono- ou multithématique selon le cas. En l'absence de définition réglementaire de ces structures en France, on peut rappeler la définition donnée par l'Organisation de Coopération et de Développement Économiques (OCDE) qui considère que les CRB sont des éléments essentiels de l'infrastructure sur laquelle s'appuient les biotechnologies et les sciences de la vie. ${ }^{[12]}$ Outre la détention de matériel biologique humain pour le cas particulier qui nous intéresse, les CRB se doivent de posséder des bases de données informatisées contenant des informations moléculaires, physiologiques et structurelles sur les collections détenues et ce dans le respect des diverses lois en vigueur. Par ailleurs, les CRB doivent satisfaire aux critères élevés de qualité et d'expertise exigés par la communauté internationale des chercheurs et des industriels en matière notamment de diffusion de l'information et de matériels

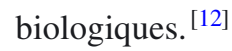

Bien que ne disposant pas d'un statut réglementaire précis, bon nombre des structures existantes, notamment publiques, ont été reconnues par le MESR à travers l'appel d'offre «Collections biologiques » de 2004 ou plus récemment à travers les procédures de déclaration d'activités de collection. Dans une démarche 
dynamique, nombre de ces structures sont par ailleurs impliquées dans des processus de certification qualité. Ces processus, en partie financés par l'Agence Nationale de la Recherche, représentent une véritable valeur ajoutée dans le cadre de l'organisation en réseau national et européen. ${ }^{[10,11]}$

\subsection{Freins au fonctionnement et au développement des CRB}

Au-delà de cette vision d'un monde potentiellement parfait, de nombreux freins persistent. Comme cela a été souligné à plusieurs reprises, notamment à l'occasion d'un rapport récent rédigé par l'Académie Nationale de Médecine, ${ }^{[10,13]}$ l'un des principaux problèmes est le manque d'un cadre législatif approprié avec pour corollaire des difficultés de financement et de pérennité. Il devient donc nécessaire que soit défini un statut des CRB afin de garantir leur autonomie et d'assurer les moyens nécessaires à l'exercice de leurs missions. Cela impose aussi de reconnaitre et de définir les métiers des CRB qui requièrent des compétences spécifiques. Enfin, comme cela a été abordé précédemment, il est temps par ailleurs de simplifier les processus réglementaires de déclaration et d'autorisation des activités de collection et de cession. ${ }^{[13]}$

Un pré-requis pour une meilleure reconnaissance des CRB est sans doute la mise en place d'outils d'évaluation et la définition de critères d'habilitation ou d'agrément. Parmi ces critères, pourraient être retenus l'existence d'une gouvernance clairement définie, l'établissement d'un processus d'assurance qualité, la visibilité et l'accessibilité des collections, un impact scientifique mesurable ainsi qu'un niveau d'activité suffisant. À ces conditions, acceptées par l'ensemble des professionnels, il pourrait être envisagée une reconnaissance nationale des CRB avec la mise en place de financements adaptés et récurrents.

\subsection{Propositions afin d'optimiser le fonctionnement des CRB}

- Définir un statut réglementaire des CRB pour une reconnaissance nationale

- Définition du cadre réglementaire des structures et des personnes (métiers);

- Établir une procédure spécifique d'habilitation;

- Définir les modalités de pérennisation des collections (en particulier dans le cas d'une défaillance d'un organisme ou d'une structure).

- Propositions de critères d'habilitation ou d'agrément

- Gouvernance clairement définie (conseil scientifique, définition des responsabilités du ou des organismes porteurs...);

- Processus d'assurance qualité reconnu et validé (certification);
- Visibilité et accessibilité des collections;

- Niveau d'activité suffisant et politique d'excellence scientifique.

- Modalités de financement

- Financement récurrent et adapté sous-tendu par une évaluation régulière et codifiée;

- Mise en place d'appels d'offre spécifiques à l'image de l'appel d'offre « Recherche translationnelle» [Direction de l'Hospitalisation et de l'Organisation des soins (DHOS), Institut National de la Santé et de la Recherche Médicale (INSERM), Institut National du Cancer (INCa)... ] ;

- Développer les financements à travers les réseaux de $\mathrm{CRB}$ [plateforme IBISA (Infrastructures en Biologie, Santé et Agronomie), Biobanking and Biomolecular Resources Research Infrastructure (BBMRI), appels d'offre européens...] ;

- Faciliter les partenariats entre les institutions publiques et l'industrie.

\section{Collections biologiques et protection des personnes}

\section{1. Implication et rôle des CPP}

En l'état actuel de la réglementation, les CPP doivent rendre un avis pour tout projet de mise en place d'une activité de collection biologique (tableau I). L'avis doit être rendu dans les 35 jours suivant la réception d'un dossier de demande complet, dossier en tout point identique à celui déposé en parallèle au ministère de la recherche. La procédure d'avis n'est pas différente, qu'il s'agisse d'une déclaration ou d'une demande d'autorisation. À ce titre, il est à noter que ni le protocole de recherche prévu à partir des collections ni même les consentements ou notes d'information ne sont envoyés au CPP. Celui-ci dispose donc d'éléments limités pour juger de la protection des personnes au regard de ces activités. Par ailleurs, l'avis est rendu au ministère de la recherche et non à l'organisme responsable de la collection. ${ }^{[7]}$

Le CPP peut également être amené à rendre un avis en cas de changement de finalité d'une collection déjà existante. Le CPP a d'ailleurs la possibilité, à titre exceptionnel et sous réserve d'une demande parfaitement motivée, de dispenser l'organisme responsable d'informer les personnes à l'origine des prélèvements. Il est en effet dit dans l'article L.1211-2 qu' «Il peut être dérogé à l'obligation d'information lorsque celle-ci se heurte à l'impossibilité de retrouver la personne concernée, ou lorsqu'un des comités consultatifs de protection des personnes, consulté par le responsable de la recherche, n'estime pas cette information nécessaire ». Au-delà de ces principes réglementaires qui pourraient paraitre simple, de nombreux problèmes pratiques restent posés. 


\subsection{Quelques problèmes particuliers}

- Consentement versus non-opposition

Par définition, le consentement est le fait de se prononcer en faveur d'un acte juridique, au sens large, et particulièrement, de toute convention, de tout contrat. Le consentement est l'élément fondamental dégagé par la doctrine de l'autonomie de la volonté : celui qui s'oblige, qui se rend débiteur d'une obligation, doit y avoir préalablement consenti. En droit de la santé, en France, ce sont les lois du 4 mars 2002 qui précisent qu' «Aucun acte médical ni aucun traitement ne peut être pratiqué sans le consentement libre et éclairé de la personne et ce consentement peut être retiré à tout moment». Le silence, en principe, ne vaut pas acceptation.

Dans le cas des collections biologiques, c'est la notion de non-opposition qui prévaut (tableau I). À ce titre, il doit donc y avoir au préalable une information de la personne. Le problème posé actuellement concerne la traçabilité de cette information et celle de la non-opposition. L'article L.1211-2 précise que «Le prélèvement d'éléments du corps humain et la collecte de ses produits ne peuvent être pratiqués sans le consentement préalable du donneur. Ce consentement est révocable à tout moment.». Seule l'utilisation de ces prélèvements doit faire l'objet d'une information et d'une non-opposition comme précisé dans ce même article ( «L'utilisation d'éléments et de produits du corps humain à une fin médicale ou scientifique autre que celle pour laquelle ils ont été prélevés ou collectés est possible, sauf opposition exprimée par la personne sur laquelle a été opéré ce prélèvement ou cette collecte, dûment informée au préalable de cette autre fin. »). Des problèmes très pratiques concernant le retrait de consentement, l'opposition a posteriori ou les modalités de l'information suite à la destruction des échantillons sont aujourd'hui posés sans réponse du législateur.

\section{- Utilisation des collections anciennes}

Rien n'est clairement spécifié au sujet de la validation des collections anciennes, celles qui par définition ont été constituées avant l'application des lois de bioéthique. Ces collections sont, pour certaines, sans garantie d'information et/ou de consentement. En toute rigueur, ces collections, si elles ne sont pas conformes à la législation actuelle, ne peuvent être utilisées. Or, certaines d'entre elles présentent un caractère scientifique non négligeable et il serait préjudiciable de devoir s'en séparer. Il demeure donc nécessaire que la loi prévoit les conditions qui permettraient de les utiliser.

- Traçabilité des consentements et retour vers la personne en cas de changement de finalité

En tenant compte du contexte et en définissant clairement les responsabilités de chaque intervenant, il est indispensable d'assurer une bonne traçabilité des consentements, afin de permettre notamment le retour vers les sujets en cas de changement de finalité. Dans le cas des recherches ne nécessitant qu'une simple information, il convient également de mettre en place une procédure permettant de retrouver facilement les personnes à l'origine des prélèvements afin le cas échéant de procéder à une nouvelle information. Néanmoins, de telles démarches peuvent être contradictoires avec la réglementation sur la protection des données personnelles.

\section{- Collections sur mineurs}

Lorsque les recherches sont menées sur des prélèvements issus de personnes mineures ou majeures sous tutelle, l'opposition est exercée par les titulaires de l'autorité parentale ou le tuteur. Se pose alors la question de l'utilisation d'anciennes collections pour lesquels les donneurs sont devenus majeurs. Doit-on procéder à une nouvelle information et dans l'affirmative, comment?

\section{- Recherche et caractéristiques génétiques}

Le cas des recherches en génétique pose également de nombreuses difficultés, au premier rang desquelles l'utilisation des prélèvements. Le code civil demande à ce que toute recherche génétique ne soit effectuée qu'après obtention d'un consentement écrit (tableau I). Se pose alors le problème de l'utilisation de prélèvements pour lesquels seul un consentement oral et/ou une information avec recherche de non-opposition sont disponibles. Compte tenu du développement de certains aspects de la biologie (génomique en particulier), comment définir ce qui relève ou non des caractéristiques génétiques, notamment ne convient-il pas de différencier les recherches génétiques identifiantes (c'est-à-dire les recherches permettant d'identifier le donneur du prélèvement) des recherches génétiques non identifiantes (celles évaluant certains éléments génétiques ne permettant pas d'identifier le donneur)? Un dernier problème dans ce contexte concerne l'éventuelle possibilité d'études sur des collections issues de donneurs décédés au moment du changement de finalité.

\subsection{Propositions émises au cours de la table ronde}

- Rôle du CPP

- Consultation des CPP pour chaque constitution de collection biologique ou lors de chaque changement de finalité d'une collection biologique existante ;

- Demande d'avis réalisée sur la base d'un dossier simplifié contenant comme pièces principales le protocole de la recherche, les documents d'information et de consentement, ainsi que les modalités d'information et de recueil du consentement des donneurs ;

- L'absence de réponse du CPP dans les 35 jours a la valeur d'un avis favorable; 
- Consultation des CPP afin de valider l'utilisation d'une collection ancienne avec impossibilité de retour aux donneurs ;

- Définir les cas où, en cas de changement de finalité, le retour vers la personne n'est pas nécessaire notamment lorsque les analyses sont effectuées après anonymisation totale ou éventuellement lorsque les échantillons biologiques proviennent de personnes décédées à la date du changement de finalité.

- Information et consentement

- Remplacer le concept de non-opposition par celui de consentement en favorisant, sans l'imposer, la signature de consentements écrits (pas seulement pour les études de génétiques);

- Optimiser les procédures de traçabilité des consentements en parallèle du recueil de données phénotypiques appropriées ;

- Conserver sur le site du prélèvement des échantillons (centre de recherche clinique, service hospitalier...) et au sein des CRB les copies des consentements écrits dans le respect des règles de l'anonymat et des référentiels CNIL.

- Cas particulier des études sur mineurs

Nous proposons 2 possibilités à discuter avec le législateur :

- Information initiale des parents sur les droits de leur enfant à sa majorité, et en particulier sur le fait qu'il pourra à tout moment revenir sur la décision prise par ses parents. Le consentement ne serait alors recueilli qu'auprès des seuls parents ;

- Demande systématique de consentement à la majorité de l'enfant. Cette $2^{\text {de }}$ solution, qui protégerait probablement mieux les intérêts de la personne, sera plus difficile à mettre en œuvre et pourrait limiter l'intérêt de certaines collections en cas du recueil impossible du consentement.

- Recherche et génétique

- Adapter les dispositions du code civil en distinguant les recherches identifiantes et non identifiantes;

- Maintien des contraintes actuelles pour les recherches génétiques identifiantes alors que pour les autres le pré-requis réglementaire serait identique à celui des collections sans analyse génétique ;

- Envisager la possibilité de recherches à visée génétique, y compris identifiantes, sur des collections anciennes lorsque le retour au sujet pour demande de consentement écrit n'est pas possible et ce après avis d'un CPP.

\section{Enjeux économiques, scientifiques et de Santé publique des collections biologiques}

\section{1. Collections et réseaux (nationaux et internationaux)}

Dans le domaine des problèmes relatifs aux enjeux économiques et scientifiques des activités de collection biologique, force est d'abord de constater la faible représentation des structures françaises dans les consortiums internationaux. Pourtant, l'intégration à de grands réseaux internationaux est un élément essentiel pour le développement de travaux multicentriques de grande ampleur et l'opportunité par ailleurs d'augmenter encore le niveau d'excellence scientifique. À l'initiative du MESR et de l'INSERM, les structures nationales (CRB) impliquées dans des activités de collection biologiques se sont rassemblées et ont entamé un travail commun dans le cadre notamment de la définition d'un référentiel en vue d'une certification. Il existe par ailleurs quelques réseaux nationaux thématisés comme c'est le cas par exemple du réseau Telethon. Un appel d'offre «Centres de Ressources Biologiques » coordonné par le MESR et le GIS «Infrastructures en Biologie, Santé et Agronomie » (IBISA) permet actuellement d'envisager une meilleure coordination nationale de l'activité de ces structures ainsi qu'un dialogue autour du problème des systèmes d'information et de leur éventuelle interopérabilité. Au sein de cet appel d'offre récent, un rapprochement avec les structures ayant une activité dans le cadre d'autres règnes biologiques (microbien, animal, végétal), notamment pour ce qui concerne les aspects qualitatifs de la conservation à long terme d'échantillons biologiques, apparait comme un progrès indiscutable. Enfin, il faut noter que des structures françaises de plus en plus nombreuses s'impliquent dans le réseau européen BBMRI, réseau qui devrait permettre à terme une meilleure lisibilité internationale des activités en matière de gestion de ressources biologiques.

Au-delà de ces aspects organisationnels, un grand nombre de problèmes pratiques subsistent néanmoins, problèmes qui sont pour certains la conséquence d'une variabilité importante de la législation en fonction des pays concernés. Il s'agit notamment de difficultés dans la gestion des procédures d'importation et d'exportation d'échantillons voire du problème de la diffusion des données associées, problème particulièrement complexe lorsque les échanges s'effectuent en dehors de l'Europe. Il existe d'ailleurs dans ce contexte une faille réglementaire dans la mesure où des échantillons biologiques immédiatement envoyés à l'étranger (où le cas échéant ils pourraient faire l'objet d'une collection) ne sont soumis qu'aux seules contraintes du transfert et non pas forcément à celles des collections. Enfin, la réglementation s'appliquant aux conditions de transport, notamment aérien, des échantillons biologiques reste aussi contraignante. L'IATA (International Air Transport Association) définit en effet très précisément les modalités du transport des échantillons biologiques. La remise à jour périodique de ces règles en augmente encore le caractère astreignant.

\subsection{Valorisation des activités de collection biologique}

En dehors de quelques exemples particuliers comme la structure BIOVALO à Lille qui regroupe des partenaires tels que 
l'Université de Lille 2, le CHU, l'INSERM et l'entreprise Eurasanté avec pour objet de devenir un guichet unique pour l'aide à la valorisation dans le domaine de la Santé (formalisation des inventions, protection de l'innovation, études de marché, montage des projets, contractualisation... ), il existe d'importantes difficultés dans la mise en place de partenariats en vu d'une valorisation des activités. Que les partenariats concernent des institutions publiques entre elles ou des interactions public/privé, il est indispensable que soient repensés les processus de valorisation entre les différents partenaires réunis autour d'un projet de recherche, notamment lorsque sont mis en jeu des échantillons biologiques et la possibilité éventuelle de développer un ou plusieurs brevets. La lourdeur des démarches administratives et parfois les querelles de clocher, notamment entre établissements publics, rendent difficile une visibilité et une compétitivité optimales au niveau international. Dans ce contexte, un décret relatif à la gestion, entre personnes publiques, de la propriété industrielle des résultats issus de travaux de recherche vient d'être publié. ${ }^{[14]}$ Ce décret prévoit notamment qu'un seul établissement soit responsable de la valorisation d'une invention, c'est-à-dire du dépôt et du suivi des brevets, et ce même si la recherche est menée par une ou des équipes ayant plusieurs tutelles publiques. Ce décret issu du rapport d'inspection sur la valorisation de la recherche publié en novembre 2006 pourrait ainsi permettre de remédier à un certain nombre des difficultés rencontrées jusqu'à ce jour.

\subsection{Propositions}

- Simplification du processus administratif d'autorisation d'importation/exportation d'échantillons biologiques humains ;

- Favoriser la structuration en réseau national des CRB et la participation à des réseaux internationaux;

- Mise en place d'outils réglementaires permettant d'améliorer les processus de valorisation en support de la recherche avec notamment le développement de structures d'aide à la valorisation et la définition de politiques contractuelles de gestion des brevets et publications.

\section{Conclusion}

Les participants à la table ronde proposent une reformulation de la loi concernant les activités de collection biologique, clarifiant notamment les rôles respectifs du MESR et des CPP et redéfinissant leur procédure de saisine. D'autre part, il semble nécessaire de mettre en place une méthodologie de référence CNIL spécifique, de préciser les procédures d'information et de consentement, en particulier dans le cadre des études de génétique et de mieux définir les modalités de fonctionnement, d'habilitation et de financement de structures dédiées aux activités de collection biologique comme les CRB. Enfin, en parallèle de la simplification des procédures relatives au dépôt de brevets, il est impératif de clarifier les modalités d'autorisation d'exportation et d'importation d'échantillons biologiques humains afin notamment de faciliter les collaborations internationales. Ces collaborations, à travers la mise en place de réseaux impliquant à la fois des structures et chercheurs publics et privés, constituent en effet un enjeu majeur tant sur le plan scientifique que sur celui de la valorisation des résultats des recherches réalisées sur des éléments biologiques issus du corps humain.

\section{Participants}

Béatrice Barraud (INSERM, Institut de Santé Publique, Pôle Recherche Clinique, Paris), Joëlle Benessiano (Hôpital Bichat, Paris), Ingrid Callies (Institut Pasteur, Paris), Henri Caplain (Sanofi-Aventis, Chilly-Longjumeau), Bruno Clément (INSERM, Rennes), Georges Dagher (INSERM, Institut de Santé Publique, Pôle Recherche Clinique, Paris), Lauren Demerville (CHU, Lille), Anne Marie Duguet (CHU, Toulouse), Catherine Durette (Hôpital Saint Louis, Paris), Dominique FigarellaBranger (CHU, Marseille), Laurence Fluckiger (AFSSAPSDEMEB, Saint Denis), Élisabeth Frija-Orvoen (Hôpital PitiéSalpêtrière, Paris), Coraline Gadras (CRB de Touraine, Tours), Patrick Gelé (Université Lille 2, Lille), Jean-Marc Laurent-Vo (DGS, Paris), François Lemaire (Hôpital Henri Mondor, Paris), Christian Libersa (Faculté de Médecine, Lille), Marie-Élise Llau (Hôtel Dieu, CHU, Toulouse), Olivier Louvet (DIRRC Île de France, Paris), Jean-Philippe Merlio (Université Victor Segalen, Bordeaux), Armelle Mijonnet (MSD-Chibret, Paris), Jean Petitpré (France Alzheimer, Douai), Alain Pierré (Servier, Courbevoie), Karen Pinachyan (MURIGENETICS, Marseille), JeanCharles Reynier (APHM, Marseille), Anabelle Sailly (CRB CHU, Lille), Pierre Schiavi (Servier, Suresnes), Tabassome Simon (Hôpital Saint Antoine, Paris), Anne Vidal (Sunnikan Consulting, Fontenay Sous Bois).

\section{Références}

1. Loi n²004-800 du 6 août 2004 relative à la bioéthique. http://www legifrance.gouv. fr

2. Décret $n^{\circ}$ 2007-1220 du 10 août 2007 relatif au prélèvement, à la conservation et à la préparation à des fins scientifiques d'éléments du corps humain et modifiant le code de la santé publique (dispositions réglementaires). http : //www . legifrance.gouv. fr

3. Loi n²004-806 du 9 août 2004 (révision de la loi Huriet) sur la protection des personnes en matière de santé. http://www. legifrance.gouv. fr

4. Décret $n^{\circ} 2006-450$ d'avril 2006 modifiant le chapitre Ier du titre II du livre Ier de la première partie du code de la santé publique relatif aux recherches 
biomédicales (dispositions réglementaires). http://www.legifrance. gouv. fr

5. Loi $\mathrm{n}^{\circ} 78-17$ du 6 janvier 1978 relative à l'informatique, aux fichiers et aux libertés. http://www. legifrance.gouv. fr

6. Loi $\mathrm{n}^{\circ}$ 2004-801 du 6 août 2004 relative à la protection des personnes physiques à l'égard des traitements de données à caractère personnel. http://www. legifrance.gouv.fr

7. Arrêté du 16 août 2007 fixant le modèle de dossier accompagnant les déclarations et les demandes d'autorisation de conservation et de préparation à des fins scientifiques d'éléments du corps humain. http://www . legifrance.gouv. fr

8. Décision du 5 janvier 2006 portant homologation d'une méthodologie de référence pour les traitements de données personnelles opérés dans le cadre des recherches biomédicales (méthodologie de référence MR-001). http://www.cnil.fr

9. Jardé O. Projet de loi sur les recherches sur la personne. Assemblée Nationale, 22 janvier 2009. http://www . assemblee-nationale.fr

10. Clément B, Grimaud JA, Deleuze JF, et al. Le réseau des centres de ressources biologiques humains. Une infrastructure essentielle pour la recherche biomédicale en Europe. Therapie 2005; 60: 351-4
11. Riegman PHJ, Morente MM, Betsou F, et al. The marble arch international working group on biobanking for biomedical research. Biobanking for better healthcare. Mol Oncol 2008; 2: 213-22

12. OCDE. Lignes directrices de l'OCDE relatives aux pratiques exemplaires concernant les centres de ressources biologique. OCDE 2007. http:// www. oecd.org

13. Hauw JJ, Ardaillou R. Modifications à apporter à la législation sur les centres de ressources biologiques. Rapport du groupe de travail de la commission 1 (biologie) de l'Académie Nationale de Médecine, 2009. http://www. academie-medecine. $\mathrm{fr}$

14. Décret $n^{\circ} 2009-645$ du 9 juin 2009 relatif à la gestion entre personnes publiques de la propriété industrielle des résultats issus de travaux de recherche réalisés par des fonctionnaires ou des agents publics. http:// www. legifrance.gouv. fr

Correspondance et offprints : Dominique Deplanque, Département de Pharmacologie Médicale, Faculté de Médecine, Centre Hospitalier Universitaire, Université Lille 2, 1 place de Verdun, 59045 Lille Cedex, France.

E-mail : dominique.deplanque@univ-lille2.fr 